Abstracta Iranica Abstracta Iranica

Revue bibliographique pour le domaine irano-aryen

Volume 32-33 | 2013

Comptes rendus des publications de 2009-2010

\title{
The Wizirgerd i Dènig and the Evil Spirit: Questions of Authenticity in Post-Classical Zoroastrianism
}

\section{Mihaela Timus}

\section{(2) OpenEdition}

1 Journals

\section{Édition électronique}

URL : http://journals.openedition.org/abstractairanica/40804

DOI : 10.4000/abstractairanica.40804

ISSN : 1961-960X

Éditeur :

CNRS (UMR 7528 Mondes iraniens et indiens), Éditions de l'IFRI

\section{Édition imprimée}

Date de publication : 1 décembre 2013

ISSN : 0240-8910

Référence électronique

Mihaela Timus, "The Wizirgerd i Dēnīg and the Evil Spirit: Questions of Authenticity in Post-Classical Zoroastrianism », Abstracta Iranica [En ligne], Volume 32-33 | 2013, document 310, mis en ligne le 01 juillet 2016, consulté le 03 octobre 2020. URL : http://journals.openedition.org/abstractairanica/40804 ; DOI : https://doi.org/10.4000/abstractairanica.40804

Ce document a été généré automatiquement le 3 octobre 2020.

Tous droits réservés 


\title{
The Wizirgerd i Dènig and the Evil Spirit: Questions of Authenticity in Post-Classical Zoroastrianism
}

\author{
Mihaela Timus
}

\section{RÉFÉRENCE}

« The Wizirgerd ì Dēnig and the Evil Spirit: Questions of Authenticity in Post-Classical Zoroastrianism », in : C. A. Bromberg, N. Sims-Williams, U. Sims-Williams, eds., BAI: Iranian and Zoroastrian Studies in Honor of Prods Oktor Skjervø, vol. 19, 2005 [2009], Detroit (Michigan), Iowa State University Press, 2009, p. 181-189.

1 En annonçant la préparation d'une édition d'un texte peu connu, le Wizirgerd i Dēnìg - Le livre des jugements religieux (WD), l'A. partage sa contribution en deux parties :

2 1. La transmission du WD au sein de la communauté parsie. Détails d'intérêt : publiée pour la première fois en 1848 (par l'Ervad Peśotan Behrāmjī Sanjānā), l'édition fut vite retirée de la circulation sous la demande de l'Anjuman parsi, fait qui explique la rareté de ce texte. Sa parution tomba au milieu des tensions entre les deux familles sacerdotales, les Sanjānā et les Bhagaria dont faisaient partie les Jāmāspāsáa. Ces derniers finirent par accuser le Dastur Edaljī Dārābjī Sanjānā (grand prêtre de Bombay et détenteur du mss. du WD selon lequel l'édition a été établie) d'avoir introduit dans le WD des passages contrevenant aux croyances et à la pratique des parsis du XIX ${ }^{\mathrm{e}} \mathrm{s}$. En particulier, le mythe des trois femmes de Zarathushtra aurait été introduit afin de justifier un acte rigoureusement prohibé : le même Dastur aurait légitimé le mariage d'un zoroastrien avec une deuxième femme alors que la première était toujours vivante. L'aberration de l'accusation s'explique par le fait que, à la même époque, une partie de la communauté parsie mettait en cause l'authenticité du Bundahišn (où l'on trouve ce mythe). Et la geste d'Ardashir (KNP), quant à elle, a été considérée comme une 
interprétation malveillante du prince fondateur. Néanmoins, si le $B d$. et le KNP ont été ultérieurement réhabilités, il n'en a pas été de même pour le WD.

3 2. Un article doctrinaire : la question de la création d'Ahreman par Ohrmazd. Bien que le WD insiste sur la position inférieure d'Ahreman, il ne manque pas de répertorier une formule qui nous était depuis longtemps familière grâce aux auteurs arabes : Ahreman créé par la pensée d'Ohrmazd (az wizir <i> Ohrmazd, WD 8.4). Au-delà de la greffe de type monothéiste de l'exégèse occidentale (d'obédience chrétienne) à l'égard du mazdéisme (Skjærvø), l'A. pense à l'impact durable de la culture islamique sur la littérature zoroastrienne "post-classique » (formule qu'il définit comme la période suivant la rédaction des principaux textes pehlevis, au IX ${ }^{\mathrm{e}}-\mathrm{X}^{\mathrm{e}} \mathrm{s}$. ap. J.-C.).

\section{AUTEURS}

\section{MIHAELA TIMUS}

Institut d'Histoire des Religions, Bucarest 